The University of San Francisco

USF Scholarship: a digital repository @ Gleeson Library | Geschke Center

2016

Better Use Case Diagrams by Using Work System Snapshots

Narasimha Bolloju

Steven Alter

Follow this and additional works at: https://repository.usfca.edu/at

Part of the Business Commons 


\title{
Better Use Case Diagrams by Using Work System Snapshots
}

\author{
Narasimha Bolloju, LNM Institute of Information Technology, Jaipur, India \\ Steven Alter, University of San Francisco, San Francisco, CA, USA
}

\begin{abstract}
Research to date shows significant variability in the success of applying the common technique of use case diagramming for identifying information system scope in terms of use cases performed by actors interacting with an information system or performed automatically by the information system. The current research tests a) the benefits of using a work system snapshot, a basic analytical tool from the work system method, before producing use case diagrams, and b) the additional benefits of enhancing use case diagramming constructs to distinguish between automated activities, activities supported by the information system, and relevant manual activities. Teams of student subjects in an experiment produced substantially better use case diagrams - containing far more use cases and qualitatively better use cases than did the teams in control group - when provided with a work system snapshot that summarized a test scenario in terms of work system concepts.
\end{abstract}

\section{KEYWORDS}

Quality Improvement, Use Case Diagrams, Work System Method, Work System Snapshots, Work System Theory

\section{INTRODUCTION}

Identifying specific processes and activities to be supported by an information system is often a daunting task in requirements determination. Too frequently, important information system functionalities are omitted or misstated (e.g., Rubinstein, 2007). According to system theorists such as Ackoff (1971, 1973), Checkland (1999), and Churchman (1970), understanding any system requires understanding of the larger system that it serves. Information systems are computerized artifacts that are used by participants in work systems. Therefore, understanding of the work system that is supported is a good starting point for understanding an information system and its significance. An understanding of the relationship between the information system and the work system needs to 
consider different types of activities to be supported or automated by the information system as part of the work system.

Use case diagrams are used widely for defining and depicting the scope of an information system in terms of a set of use cases corresponding to activities supported or automated by the information system. Although there is no totally standard definition of use case, there are several working definitions such as the following (OMG, 2010 - p 628):

A use case is the specification of a set of actions performed by a system, which yields an observable result that is, typically, of value for one or more actors or other stakeholders of the system.

At the beginning of an attempt to identify use cases within a particular work system, it is often unclear what the boundaries of the information system will be, i.e., which activities will be automated or supported by the information system, and which activities are part of the work system but will not be automated or supported directly by the information system. Consequently, the specification of use cases within a use case diagram created at the beginning of an object-oriented analysis should start with identification of the work system and then should identify different types of activities.

A good approach for doing this is to summarize the ("as is" or "to be") work system using a work system snapshot (a basic analytical tool from the work system method (Alter, 2006; 2008; 2013), and then to delve more deeply into each of the activities within the work system snapshot, to identify use cases to be automated or supported by the information system, and then to look at them in more depth to clarify specific functionality required in the information system (see Appendix 1 for a brief introduction to the work system framework and work system snapshots). Thus, our proposed approach recommends summarizing the business situation using a work system snapshot before developing use case diagrams. For this purpose, we propose enhancements to use case diagramming constructs to distinguish between work system and information boundaries, and to differentiate various types of use cases. Evidence from the current research implies that producing a work system snapshot before developing use case diagrams may help analysts identify more use cases that are also of higher quality, thereby help in better determination of the scope and function of the information system to be developed.

This paper proceeds as follows. First it provides background about scope determination in systems analysis, use case models, the work system method, and challenges associated with use case diagrams. Next it presents the proposed approach for improving the results of use case diagramming by using work system snapshots. It reports on a classroom experiment in which teams of student subjects created use case diagrams for a case study involving a university book store. The teams in the control group used traditional use case diagramming. In the first experimental treatment, the teams produced use case diagrams after seeing a work system snapshot that summarized the situation. In the second experimental treatment, the teams also saw the work system snapshot, but then produced enhanced use case diagrams that included explicit boundaries between non-supported activities, supported activities, and totally automated activities. Analysis of the results shows that the beneficial impact of both treatments is statistically significant. The conclusion discusses possible implications for research and practice.

\section{BACKGROUND}

At the outset, it is important to note that this paper distinguishes between two types of systems:

- Work systems are systems in which people and/or machines use information and technology to produce product/services for internal or external customers. (Alter, 2013). By default, human participants are viewed as part of a work system rather than as users of a work system. 
- Totally automated information systems are computerized artifacts (hardware and software) that are used by users as they participate in work systems.

In computer science, and in most discussions of use cases, the term information system is assumed to refer to totally automated information systems, i.e., one or more computerized artifacts (the second definition) rather than work systems with human participants. In contrast, most discussions of systems in organizational behavior and management assume explicitly or implicitly that people may be essential elements of the system of interest, rather than just users of computerized artifacts.

Part of the difficulty in determining information system requirements is related to confusions about whether "the system" is a work system with human participants or a totally automated information system with human users. For the sake of clarity and internal consistency within this paper and in relation to literature related to use cases, this paper uses the computer science view of the term "information system". I.e., the term refers to totally automated information systems. In contrast, it uses the term "work system" to refer to systems in which people are participants. Most work system research, including research mentioned in Alter (2013) does not use the computer science view of information system. Instead, it treats the concept of information system as a special case of work system, i.e., a work system in which all of its processes and activities are devoted to information processing activities such as creating, transmitting, storing, retrieving, manipulating, and displaying information.

\section{Determination of Scope}

The importance and difficulty of determining the proper scope of an information system in a systems analysis effort have been discussed many times. Nuseibeh and Easterbrook (2000, p44) point out that "requirements modeling and analysis cannot be performed adequately in isolation from the organizational and social context in which any new [information] system will have to operate." Inherent difficulties in requirements determination are related to the nature of expected information system functionality, decisions about the information system's boundary, environmental conditions, and understandability and usability of resulting artifacts (Cheng \& Atlee, 2007). In proposing a goaloriented analysis technique, Mylopoulos et al (2001) highlight the need for exploring alternatives for achieving organizational and technical objectives prior to elaborating those requirements during subsequent phases. Since the information system's scope defines the functionality to be provided, under-scoping or over-scoping an information system can lead to architectures with inappropriate functionality (Buschmann, 2009).

Identifying various activities performed in the present work system (see Appendix) is a reasonable starting point for deciding which activities must be included in the scope of the information system being developed. Figure 1 depicts different types of activities and shows that activities may be linked to form processes in a typical work system. The distinction between work systems and information systems (as defined above) implies that four types of activities might be relevant when defining the scope of information systems that support specific work systems:

1. Automated Activity: An activity within a work system is totally automated by the information system. It does not require human actors.

2. Directly Supported Activity: An activity within a work system is performed by an actor (participant) who uses the information system interactively to perform the work. Using an information system interactively refers to using the information system through a user interface that can receive requests and other information from the user and/or can provide information to the user.

3. Indirectly Supported Activity: An activity within a work system is performed by an actor (participant) who does not use the information system interactively to perform the work, but it 
Figure 1. Processes and activities in a typical work system

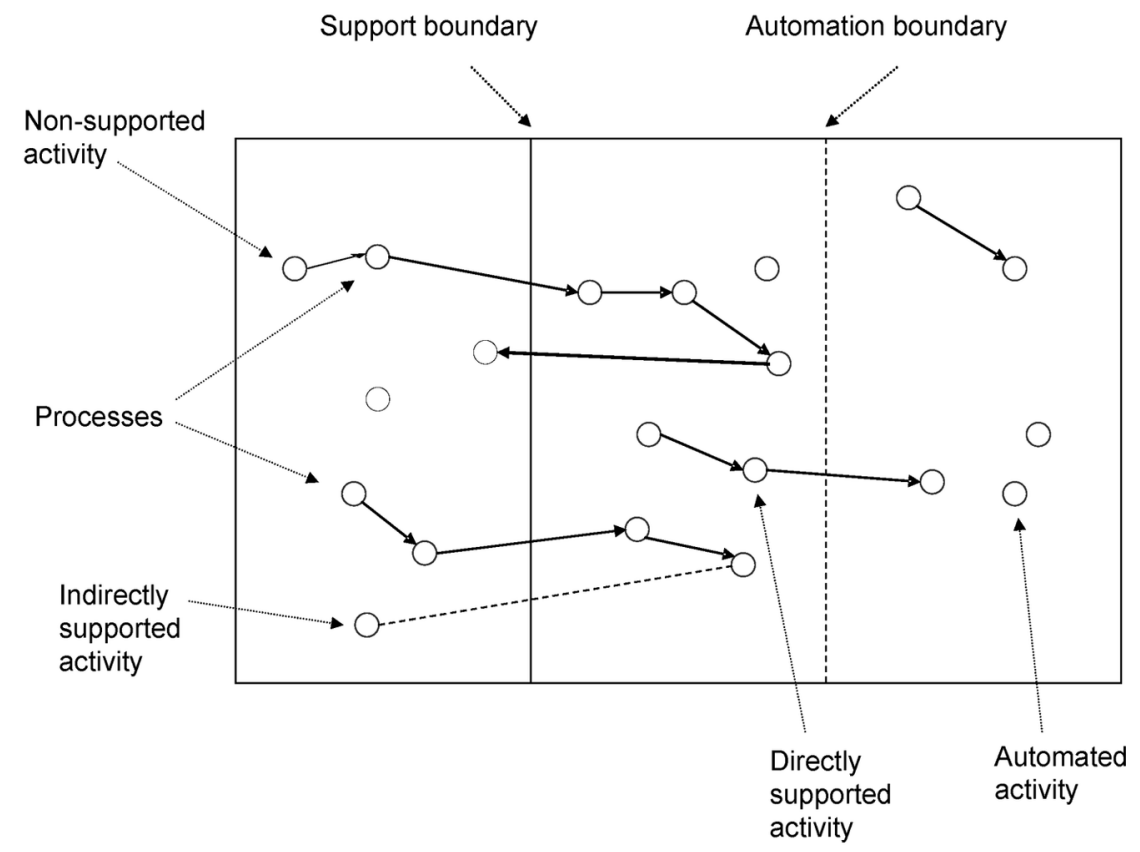

is supported indirectly by the information system, such as by receiving an analysis that someone else within the work system produced by using the information system directly.

4. Non-Supported Activity: An activity within a work system is performed without support by the information system. That activity is still part of the work system, and therefore is relevant for understanding the context of the information system.

While an understanding of the situation (and hence the analysis) includes four types of activities, only the first and second types are within the scope of the (computerized) information system.

\section{Use Case Diagrams}

The Unified Modeling Language (UML) has been used successfully in creating integrated representations that serve the development community in defining detailed software requirements. Many experts (e.g., Rosenberg \& Stevens, 2007) suggest a use case driven approach, where use case models are developed to determine general requirements which form the basis for producing other artifacts such as class diagrams and sequence diagrams. Use case models are composed of two types of artifacts: a) use case diagrams identifying use cases to be supported and providing links to actors involved in those use cases, and b) use case narratives explaining the use cases in more detail by summarizing the functionality provided by the information system and by explaining how the actors interact with the information system. Although a single use case diagram suffices for simpler information systems, most real world systems require a set of use case diagrams.

Although the pictorial representation of use case diagrams is easier to understand, the task of determining the required information system functionality in terms of use cases is challenging because the information system's scope and functionality are not obvious in advance. Ideally, use case diagrams should be complete and their use cases should be valid. Completeness refers to inclusion of all use cases that should be supported by the required information system functionality. Validity refers to exclusion of unnecessary or irrelevant functionality. A high quality use case diagram should include 
all the use cases that should be supported by required functionality, and should not contain use cases that involve unnecessary functionality or non-supported activities.

Producing high quality use cases is difficult, especially for novice systems analysts. For example, an empirical study on the quality of some commonly used UML artifacts (Bolloju \& Leung, 2006) reported that more than half of the use case diagrams contained "manual operations listed as use cases". Our proposed use of a work system snapshot in the process of use case diagramming is designed to improve both completeness and validity. Previous attempts to support use case modeling include a checklist-based inspection technique for detecting typical defects (Anda \& Sjoberg, 2002) and application of coordination analysis based on coordination theory and dependency diagrams (Liu \& Wyner, 2009). In conclusions from earlier research on problems and deficiencies of UML for requirements specification, Glinz (2000) argues that use case constructs should be extended to support notifications to external actors, interactions between actors and decomposition of complex use cases.

Commenting on the practical limitations of use case models, Baekgaard (2005) notes unrealistic assumptions that the border between the IT-system and its environment is clear and that activities of actors are well-understood and can be reduced to interaction with the IT system. Kim et al. (2006) argues that use-case driven analysis does not provide an adequate rationale for the various artifacts generated during the requirements analysis. Rational Software published an article about correcting ten ways in which project teams misuse use cases (Gottesdiener, 2002).

\section{The Work System Method}

The work system approach for understanding and analyzing work systems from a business perspective (Alter, 2006; 2013) starts from a different viewpoint and addresses some of the issues that use case modeling does not address well. The work system framework (see Appendix) helps in painting a broader picture that includes human participants, product/services that are produced, customers, the surrounding environment, infrastructure, and relevant strategies. As illustrated by Table 1, one of the primary tools in the work system method for understanding and analyzing systems in organizations is a work system snapshot, which summarizes a work system efficiently and effectively in terms of six of the nine elements of the work system framework. In contrast to the way a use case diagram identifies activities that "use" the information system and other relevant activities that do not use the information system, a work system snapshot does not start with a distinction between the work system and the information system that supports it. Instead, it assumes that the work system simply uses certain software and hardware that is listed under technologies. The boundaries of the information system are determined later in the analysis, after the scope of the work system is clarified.

Work system snapshots offer a simple mechanism for summarizing a business situation and facilitating deeper analysis of the views of different stakeholders. Our research examines the impact of incorporating a work system snapshot into the process of creating a use case diagram during requirements determination, bridging differences in system scope between developer and stakeholders' interests and perspectives because of better identification specific activities to be supported by the system. Work system snapshots, apart from being reasonably easy to use, were found to be playing a central role in verifying internal consistency (Truex et al., 2011). Our approach of employing work systems snapshot as an intermediate artifact would help in clarifying thoughts of analysts before producing use case diagrams.

\section{ENHANCED USE CASE DIAGRAMMING}

In addition to testing a potential improvement in the process of use case diagramming, our research also tests an enhancement to the use case diagrams themselves. The proposed enhancement has three parts: 
Table 1. An example of work system snapshot

\begin{tabular}{|c|c|c|c|}
\hline \multicolumn{2}{|c|}{ Customers } & \multicolumn{2}{|c|}{ Product/Services } \\
\hline \multicolumn{2}{|c|}{$\begin{array}{l}\text { - Hiring manager } \\
\text { - Larger organization (which will have the applicant as a } \\
\text { colleague) } \\
\text { - HR manager (who will analyze the nature of applications) }\end{array}$} & \multicolumn{2}{|c|}{$\begin{array}{l}\text { - Applications (which may be used for subsequent } \\
\text { analysis) } \\
\text { - Job offers } \\
\text { - Rejection letters } \\
\text { - Hiring of the applicant }\end{array}$} \\
\hline \multicolumn{4}{|c|}{ Major Activities and Processes } \\
\hline \multicolumn{4}{|c|}{$\begin{array}{l}\text { - Hiring manager submits request for new hire. } \\
\text { - Staffing coordinator defines the parameters of the new position. } \\
\text { - Staffing coordinator publicizes the position. } \\
\text { - Applicants submit resumes. } \\
\text { - Staffing coordinator selects shortlisted applicants and sends the list to the hiring manager. } \\
\text { - Hiring manager identifies applicants for interview by browsing applicant resumes. } \\
\text { - Staffing coordinator sets up interviews. } \\
\text { - Hiring manager provides feedback from the interviews. } \\
\text { - Staffing coordinator or staffing assistant sets up additional interviews with other employees. } \\
\text { - Hiring manager makes hiring decisions. } \\
\text { - Staffing assistant sends offer letters or rejections. } \\
\text { - Successful applicant accepts or rejects job offer. }\end{array}$} \\
\hline Participants & \multicolumn{2}{|c|}{ Information } & Technologies \\
\hline $\begin{array}{l}\text { - Hiring managers } \\
\text { - Staffing coordinator } \\
\text { - Applicants } \\
\text { - Staffing assistant } \\
\text { - Other employees who perform } \\
\text { interviews }\end{array}$ & \multicolumn{2}{|c|}{$\begin{array}{l}\text { - Job description } \\
\text { - Advertisements } \\
\text { - Job applications } \\
\text { - Cover letters } \\
\text { - Applicant resumes } \\
\text { - Short list of applicants } \\
\text { - Information and impressions from the } \\
\text { interviews } \\
\text { - Formal documentation of the } \\
\text { interviews. } \\
\text { - Job offers } \\
\text { - Rejection letters }\end{array}$} & $\begin{array}{l}\text { - Word processor } \\
\text { - XYZ HR database software } \\
\text { - Telephones } \\
\text { - Email }\end{array}$ \\
\hline
\end{tabular}

1. Representing in the diagram both the information system and the work system that is supported. This is accomplished by including a work system boundary that encloses the information system boundary.

2. Including totally automated use cases that do not involve human actors as users. This enhancement addresses the possibility of not associating any actor with use cases representing automated activities. Traditional use case diagrams employ actors such as "time" to indicate such use cases. By not linking use cases of this type to any actor, it is possible to represent various types of automated activities (e.g., use cases triggered by certain attribute values or conditions).

3. Including indirect and non-supported activities to provide a better understanding of the context. This is accomplished by using dotted lines for linking use cases with supporting actors.

The proposed enhancements are depicted in Figure 2, which represents a generic use case diagram. This diagram includes use cases corresponding to automated activities, and activities directly or indirectly supported by the information system and non-supported activities. Although typical use cases represent activities that include steps performed by involving human actors and the information system, completely automated use cases do not have any steps performed by human actors. As part of enhancements to use case diagramming, we extend use cases to include relevant activities performed completely by human actors without direct use of the information system. By including such use 


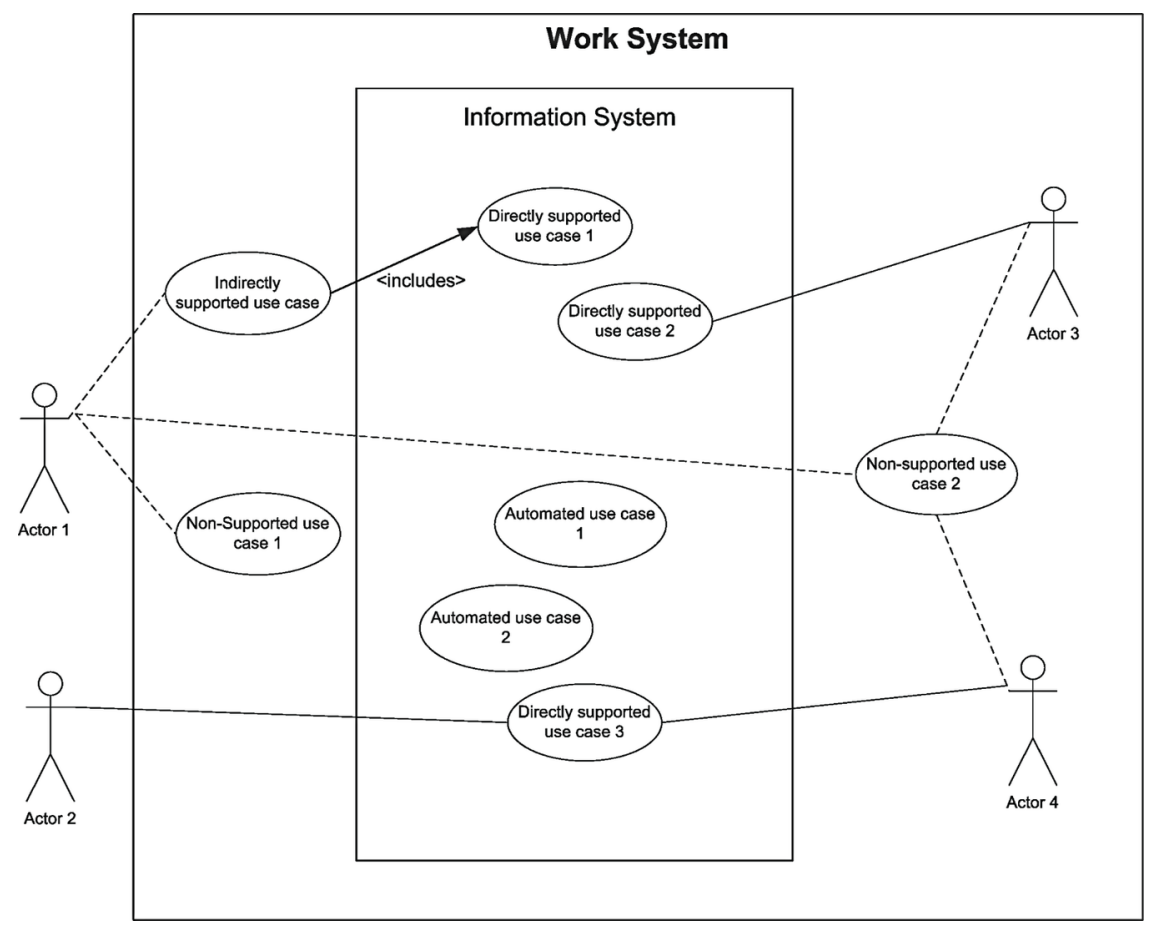

cases in the diagram explicitly, we believe that erroneous depiction of manual activities as use cases within the information system could be avoided. Indirectly supported use cases are connected to the actor with a dotted-line and connected to some supported use cases with an include link.

Figure 3 is an example of an enhanced use case diagram corresponding to the "Hiring New Employees," scenario, which was summarized by the work system snapshot shown in Table 1 . The use cases to be supported by the information system (e.g., submit resumes, select shortlisted applicants) and those completely automated (e.g., send rejection letters) are enclosed within the information system boundary. The remaining use cases (e.g., publicize the position and setup interviews) represent activities that are indirectly supported by the information system.

Figure 3 separates activities to be supported by the information system (e.g., submit request for new hire and submit resumes) and activities to be automated (e.g., send rejection letters) from the activities that are performed without direct use of the computerized information system (e.g., identify applicants and making hiring decisions). It is, however, possible that certain activities belonging to the work system may make use of some of the intermediate products and/or informational entities. For example, the activity makes hiring decisions use applicants' resumes and feedback.

In addition, the actors are located inside and outside the work system boundary to differentiate work system participants from work system customers who are not participants. For example, actors representing participants (e.g., Staffing coordinator) and customers (e.g., Applicants) are placed inside and outside the work system boundary respectively. The dotted lines between use cases and actors indicate the supporting role of the linked actor in completing the activities pertaining to the use case. For example, the use case "schedule additional interviews" performed by the Staffing Coordinator requires consultation support from Hiring Manager, Shortlisted Applicants, and Other interviewers. 


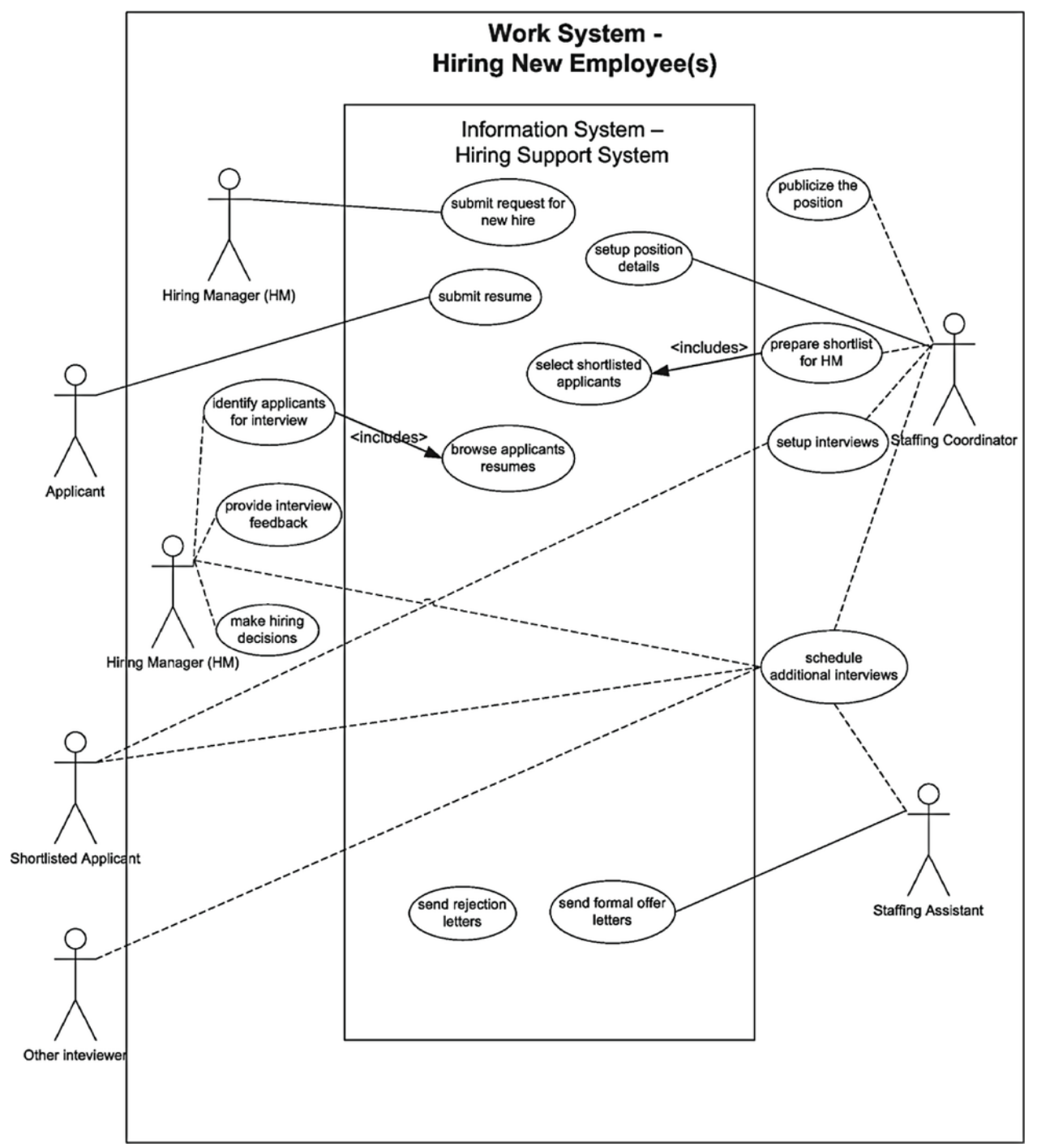

\section{Creating an Enhanced Use Case Diagram from a Work System Snapshot}

The overlap between work system snapshots and corresponding enhanced use case diagrams implies a procedure for creating an enhanced use case diagram from a work system snapshot:

Step 1: Map each activity or process in the work system snapshot to one or more use cases and place those use cases either inside the information system boundary or outside the information system boundary. It is possible to decompose and represent certain complex activities and processes with two or more corresponding use cases.

Step 2: Map each participant to an actor inside the work system boundary. Note that some customers may also be work system participants (e.g., in self-service work systems and in many other service situations such as personal services). Map non-participant customers to actors outside of the work system boundary. 
Step 3: Associate each actor to the use cases performed by that actor, and link each use case to supporting actors, if any. (Use cases that are completely automated are not associated with any actor).

Step 4: Refactor use cases using generalize, include and extend relationships. Refactoring refers to revisions such as fragmenting complex use cases into simpler ones to establish include and extend types of relationships, and creating generic use cases from a set of use cases sharing significant common functionalities.

Step 5: Review and relocate the use cases that represent indirectly supported or non-supported activities within the work system boundary but outside of the information system boundary. Also, make sure that the use cases that can be supported by the information system are within the information systems boundary

Step 6: Write a brief description for each use case identified.

\section{THE EXPERIMENT}

We conducted a laboratory experiment to study the effectiveness of creating a work system snapshot before producing use case diagrams. Student subjects produced use case diagrams for the University Bookstore Limited (UBL) case, which had been used several times in prior classes. The UBL case is a 3-page textual description of a situation that includes selling books to customers, processing textbook adoption requests by faculty, pre-orders for books from students, and ordering of books from publishers and distributors. In addition, the UBL case included 5 pages of sample documents used in that situation.

\section{Subjects}

The subjects were second year undergraduate (BBA) students majoring in information systems or electronic commerce at a major Asian university. These students were in 5 tutorial groups (each group with about 30 students). The academic background and work experience of these full-time students was quite similar (age 19-21 years, very little work experience, and completion of 3 or 4 introductory level courses on information systems).

\section{Task}

Performed in teams of size 3 or 4 , the task was to develop a use case diagram (either simple or enhanced) for the UBL case study in a two-hour laboratory session using a work system snapshot prepared by the instructor. A week prior to the experiment the subjects received a brief introduction to the work system method and then prepared work system snapshots for the same case as an exercise. During this exercise, the students reviewed the UBL case study material and identified various elements required for completing a work systems snapshot. For control purposes, the instructor prepared a consolidated work system snapshot (shown in Appendix 2) rather than asking the student teams to make use of work system snapshots produced by them. In this study, we assume that the task outcome by each team of students is roughly equivalent to that of a novice systems analyst.

\section{Control}

- TRA: Traditional use case approach (existing method and existing product): Serving as a control were UBL use case diagrams produced by students in the course in the previous year (similar teams in a similar setting using a system feature list shown in Appendix 3). The students mapped features in the list to use cases and then identified relevant actors for each of use case. Since this was a teaching situation, we believed it was inappropriate to create a new control group by teaching some sections using what we thought was an improved approach and teaching other sections using what we believed was a less powerful approach. 


\section{Experimental Treatments}

- SIM: Simple use case diagramming approach (new method and existing product): Student teams in two tutorial sections produced use case diagrams using the UBL case material plus a work system snapshot supplied by the instructor. These teams followed part of the mapping procedure in section 3.1 and identified use cases to be supported by the information system. They did not perform the steps related to the work system boundary and identification of use cases corresponding to non-supported activities.

- ENH: Enhanced use case diagramming approach (new method and new product): Student teams in three tutorial sections produced enhanced use case diagrams using the UBL case material plus a work system snapshot supplied by the instructor. They followed the entire mapping procedure in section 3.1.

During the development of use case diagrams - which also required brief descriptions of use cases identified - the teams following the TRA approach referred to the feature list and the case study material, and the teams following SIM and ENH approaches referred to the work system snapshot, and the case study material.

\section{Inputs Provided}

- UBL case study

- For student teams following TRA approach, a one-page list of required information system features, presented in Appendix 3, for conducting business and features for analyzing business (Coad et al., 1995). This list of information system features was consolidated by the instructor based on the draft versions produced by the student teams in an earlier session following a procedure similar to that followed by SIM and ENH treatment teams.

- For student teams following SIM or ENH approaches, the work system snapshot for the work system at UBL. The instructor prepared this consolidated work system snapshot based on snapshots produced by all the same student teams in an earlier session (shown in Appendix 2).

The inputs provided to the teams following different approaches is identical except for the intermediate artifacts as described above.

\section{Output Collected}

Use case diagrams created by the student teams using the ArgoUML case tool (Ramirez et al., 2007) included descriptions of use cases that were identified. The control group is six student teams from the previous year. Each team produced use case diagrams with brief descriptions prepared for the UBL case study by student teams from the previous year.

The experiment addressed two research questions, both of which are directly related to determining the appropriate scope of an information system:

1. Does the usage of a work system snapshot result in creating more complete and valid use case diagrams?

2. Will the enhanced use case diagramming approach help in better separation of use cases corresponding to activities either automated or supported by the information system and other types of activities that should be included in an understanding of the situation?

We examine these research questions through three hypotheses, of which the first is most important and encompassing, whereas the others focus on specific components of the larger phenomenon. 
H1: Work system snapshots help systems analysts in creating more complete use case diagrams.

$\mathrm{H} 1$ addresses whether the scope of requirements defined by the set of use cases identified in the solution matches with the expected solution. Completeness of a use case diagram is measured by the percentage of required use cases found in the diagrams prepared by the analysts.

Two sub-hypotheses related to $\mathrm{H} 1$ are:

- H1a -Systems analysts using either the SIM or ENH approach will identify more required use cases compared to systems analysts using the TRA approach.

- $\quad \mathrm{H} 1 \mathrm{~b}-$ Systems analysts using the ENH approach will identify more required use cases compared to systems analysts using the SIM approach.

H2: Work system snapshots help systems analysts in creating use case diagrams with fewer invalid use cases.

This hypothesis addresses the presence of use cases that are inappropriate for the given problem domain. The relevant measure of a use case diagram is the percentage of invalid or out of scope use cases among all the use cases identified by the analysts.

Two sub-hypotheses related to $\mathrm{H} 2$ are:

- $\quad \mathrm{H} 2 \mathrm{a}$-Systems analysts using either the SIM or ENH approach will identify fewer invalid use cases compared to systems analysts using the TRA approach.

- $\quad \mathrm{H} 2 \mathrm{~b}$-Systems analysts using the ENH approach will identify fewer invalid use cases compared to systems analysts using the SIM approach.

H3: Work system snapshots help systems analysts in minimizing incorrectly located use cases.

This hypothesis attempts to verify whether the approaches involving a work system snapshot result in representing fewer non-supported activities as supported activities and fewer supported activities as non-supported activities when analysts produce enhanced use case diagrams. For use case diagrams created following TRA and SIM approaches, a use case is considered improperly located if it includes an unsupported or indirectly supported activity. For the diagrams created following ENH approach, use cases that are shown outside the information system boundary even though they can be supported or automated by the information system are also considered incorrectly located.

Two sub- hypotheses related to $\mathrm{H} 3$ are:

- H3a -Systems analysts using either the SIM or ENH approach will locate fewer use cases incorrectly compared to systems analysts using the TRA approach.

- $\quad \mathrm{H} 3 \mathrm{~b}-$ Systems analysts using the ENH approach will locate fewer use cases incorrectly compared to systems analysts using the SIM approach.

\section{Coding Scheme and Procedure}

For assessment of completeness and validity of use case diagrams, we used three expected solutions of use case diagrams (one for TRA control and one each for SIM, and ENH treatments) which included brief descriptions of use cases. These expected solutions were matched against the use case diagram and brief use case descriptions submitted by student teams as their solutions. A research assistant (RA) who had completed graduate studies received the case study material and expected solutions. One of the authors of this paper and the RA independently assessed three randomly selected student team solutions (one of each type for a total of 38 use cases) by identifying whether each use case 
in the expected solution is included in the student team solution. As part of assessing completeness (i.e. presence of required use cases), differences between expected solution and student solution were resolved after taking into account the associated descriptions. Thus, variations such as aggregation of two or more use cases in the expected solution as a single use case (treated as the specific student solution correctly combined those use cases), and decomposition of a single use case from the expected solution as multiple use cases (regarded as the required use case is correctly represented) were resolved. However, any use case found in the student solution that has no corresponding use case in the expected solution was counted as a single invalid use case. Following up on a substantial agreement between the two assessors on the numbers of required and invalid use cases in the selected student solution (Cohen's Kappa $=0.679, \mathrm{p}<0.001$ ), the RA continued with the assessment of remaining solutions.

The procedure used for assessment of use case type is based on the brief use case descriptions specified by the student teams. One of the authors of this paper and a final year undergraduate student helper ( $\mathrm{SH}$ ) independently assessed three randomly selected solutions (one of each type) by classifying each use case inside the information system boundary into one of the three categories: non-supported or indirectly supported activity, supported activity, and automated activity. No such classification was made for use cases with missing or incomplete descriptions. The inter rater reliability results indicated moderate agreement (Cohen's Kappa 0.494, p < 0.001). The SH continued with the assessment of the remaining solutions. We believe that the $\mathrm{SH}$, who had completed the systems analysis course that used the UBL case study while in a previous cohort, was more able to perform this assessment than the RA.

\section{RESULTS AND DISCUSSION}

Table 2 summarizes findings for the three approaches (TRA, SIM, and ENH) in terms of the number of use cases identified, percentage of required and invalid use cases, and percentage of incorrectly located use cases. The percentage of required use cases identified is computed based on the number of use cases in the expected solution. The other two percentages in last two columns are computed by comparing the number of invalid or incorrectly located use cases to the number of use cases in a given student team's solution.

We found that introducing the work system snapshot before producing the team's use case diagram generated twice as many use cases (8.7 for TRA vs. 17.4 for SIM and 19.6 for ENH). This result provides evidence of the benefit of using a work system snapshot before producing detailed, relatively more complete, use case diagrams.

Each of the hypotheses was tested first for differences between TRA and WSS-based use case diagramming (produced using SIM or ENH), and then for differences between SIM and ENH. The results of these tests are discussed below.

Table 2. Summary of findings for the three approaches

\begin{tabular}{|l|l|l|l|l|}
\hline \multicolumn{1}{|c|}{ Approach } & $\begin{array}{c}\text { use cases identified - } \\
\text { mean (std dev) }\end{array}$ & $\begin{array}{c}\text { \% of required use } \\
\text { cases identified - } \\
\text { mean (std dev) }\end{array}$ & $\begin{array}{c}\text { \% of invalid use cases } \\
\text { identified - mean (std } \\
\text { dev) }\end{array}$ & $\begin{array}{c}\text { \% of incorrectly } \\
\text { located use cases - } \\
\text { mean (std dev) }\end{array}$ \\
\hline $\begin{array}{l}\text { TRA } \\
(n=6)\end{array}$ & $8.67(2.73)$ & $43.8(16.77)$ & $31.94(13.44)$ & $4.63(7.38)$ \\
\hline SIM $(n=18)$ & $17.39(5.24)$ & $72.57(7.47)$ & $26.96(12.95)$ & $19.58(6.46)$ \\
\hline ENH $(n=16)$ & $19.63(3.81)$ & $82.42(11.00)$ & $33.81(13.45)$ & $11.21(6.33)$ \\
\hline WSS $(n=34)$ & $18.44(4.69)$ & $77.21(10.42)$ & $30.18(13.44)$ & $15.64(7.52)$ \\
\hline
\end{tabular}

Note: WSS = SIM + ENH (Both treatments start with a work system snapshot) 


\section{Results for Hypothesis H1}

As shown in Table 3, the average number of use cases in use case diagrams created using work system snapshot (an average of 17.39 for SIM and 19.83 for ENH) was twice as many as the average number as produced by TRA (8.67). The test of H1a shows that the difference in the percentage of required use cases ( $72.57 \%$ for SIM and $82.42 \%$ for ENH) was almost twice as high as the percentage of required use cases for TRA (43.8\%). The difference is significant at the .001 level.

Furthermore, the average of 19.63 use cases in use case diagrams created using the ENH approach was higher than the average of 17.39 use cases for SIM. Possibly more important, the test of $\mathrm{H} 1 \mathrm{~b}$ shows that the percentage of required use cases was significantly higher $(\mathrm{p}<0.01)$ with the ENH approach (82.42\%) vs. the SIM approach (72.57\%). In other words, including the information system and work system boundaries in the enhanced use case diagram helped the analysts visualize the situation more effectively.

Since both the sub-hypotheses are supported, we conclude that work system snapshot helped in creating more detailed and more complete use case diagrams. Furthermore, the ENH approach helped in creating more complete use case diagrams than were created using the SIM approach.

\section{Results for Hypothesis H2}

Hypothesis $\mathrm{H} 2$ was not supported. The results show no significant difference in mean percentage of invalid use cases. None of the three approaches exhibited superiority in preventing student teams from identifying invalid use cases.

\section{Results for Hypothesis H3}

The TRA approach produced a much smaller percentage of incorrectly located use cases than work system snapshot-based approaches $(\mathrm{p}<0.01)$. A likely explanation for this anomaly is that the TRA approach produced less than half as many use cases as the SIM and ENH approaches, thereby providing many fewer opportunities for incorrectly locating a use case. The sheer number of use cases in the SIM and ENH use case diagrams made those diagrams substantially more elaborate, with several additional use cases than those produced by the TRA approach, and therefore may have increased the likelihood that a higher percentage of SIM and ENH use cases would be located incorrectly.

As expected, the ENH approach generated significantly fewer incorrectly located use cases than the SIM approach ( $\mathrm{p}<0.001)$. This outcome is probably due to the separation of boundaries (information system and work system) in the ENH approach, which may have forced student teams to reason more carefully about the location of use cases.

Table 3. Hypotheses test results

\begin{tabular}{|l|l|l|l|}
\hline \multicolumn{1}{|c|}{ Hypothesis } & \multicolumn{1}{c|}{ Mean difference } & \multicolumn{1}{c|}{ t-stat } & \multicolumn{1}{c|}{ Result } \\
\hline H1a: & Required_WSS - Required_TRA & $6.59 * * *$ & Supported \\
\hline H1b & Required_ENH - Required_SIM & $3.09 * *$ & Supported \\
\hline H2a & Invalid_WSS - Invalid_TRA & -0.29 & Not supported \\
\hline H2b & Invalid_ENH - Invalid_SIM & 1.51 & Not supported \\
\hline H3a & $\begin{array}{l}\text { Incorrectly Located_WSS - } \\
\text { Incorrectly Located_TRA }\end{array}$ & $3.32 * *$ & Not supported \\
\hline H3b & $\begin{array}{l}\text { Incorrectly Located_ENH - } \\
\text { Incorrectly Located_SIM }\end{array}$ & $-3.87 * * *$ & Supported \\
\hline
\end{tabular}

Notes: WSS = SIM + ENH (Both treatments start with a work system snapshot);

${ }^{* *} p<0.01,{ }^{* * *} p<0.001$ 


\section{RELATED WORK}

Use case models include use case diagrams which depict the expected system functionality and use case narratives which elaborate the use cases by specifying how the actors interact with the information system. 74\% of respondents in a survey of UML practitioners (Dobing \& Parsons, 2006) indicated that they found use case diagrams useful for verification of system requirements with clients. They also reported that usage of use case diagrams ranked second, after use case narratives, with respect to client involvement in UML components. Erickson \& Siau (2007) have also found in another practitioner survey that use case diagrams ranked second among UML artifacts. On the contrary, Dobing \& Parsons (2008) have also noticed that system developers often avoid using use case diagrams and narratives, and recommend further work in modifying UML diagrams for effective communication and verification.

Gemino \& Parker (2009) reported that supplementing use case diagrams with textual descriptions results in achieving better understanding in persons viewing the use case narratives. In a similar study by Mustafa (2010) observed that provision of diagrams along with use case narratives resulted in improved comprehension performance, and also found that it did not matter whether simple or detailed use case diagrams are used for this purpose.

Considering challenges in effective use of use case diagrams and narratives, researchers and practitioners have been working on different approaches to assist systems analysts. From the practitioner perspective, approaches included structuring use cases with goals (Cockburn, 1998), offering guidelines for writing effective use cases (Cockburn, 2001), clarifying confusion between business use cases and system use cases between business analysts and system developers (Langlands \& Edwards, 2009). Approaches by researchers included extensions such as enhancing use case narratives to long lived embedded software systems (Eriksson et al., 2005), accommodating features for human actions, differentiating current system boundary with future system boundary and attaching different types of documents with interactions (Yang-Turner \& Lau, 2011), and automatic layout of use case diagrams for better visualization (Eichelberger, 2008).

Our study can be positioned as extension to use case diagramming with constructs to differentiate human activities from system activities so that assistance can be provided in determining the scope of information system under development. In addition, our method offers guidance through an intermediary artifact - the work system snapshot - which facilitates identification of relevant activities. Although our proposed extension to use case diagrams results in relatively more complex diagrams, the increased complexity is unlikely to affect comprehension and thereby may contribute to effective communication between end users and system developers.

\section{CONCLUSION}

Our experiment addressed two research questions related to determining the appropriate scope of an information system:

1. Does the usage of work system snapshots result in creating more complete and valid use case diagrams?

2. Will the enhanced use case diagramming approach help in better separation of use cases corresponding to activities either automated or supported by the information system and other types of activities that should be included in an understanding of the situation?

The strong statistical results for H1 suggests that the usage of the work system snapshot prior to developing use case diagrams may help systems analysts create more complete use case diagrams. Furthermore, the work system boundary introduced as part of enhanced use case diagramming contributes toward solving the problem of non-supported or indirectly supported activities being 
represented as supported activities that require information system functionality. The findings from the experiment demonstrate that student subjects using a work system snapshot produced substantially higher quality use case diagrams. The enhanced use case diagramming constructs contributed additional improvements in that direction. The results highlight the potential value of our proposed approach in supporting activities related to determination of the scope of information systems.

The findings from this study point to important possibilities for developing improved requirements analysis methods. While use case diagrams are widely taught and used, they have a number of previously mentioned shortcomings as a tool for identifying information system requirements when using object-oriented analysis and design methods, The incorporation of the work system snapshot into the process of creating use case diagrams addresses some of the issues cited by Gelbard et al (2010), who argue that the analysis phase should focus on organizational interactions, data items, business processes and user experience.

If validated by testing in other settings, these initial results could have important implications for improving requirements analysis processes in practice. Use of work system snapshots as part of the process of developing use case diagrams might help systems analysts visualize the business situation more fully before creating use case diagrams. Since use case diagrams are easier to comprehend compared to other UML artifacts such as class diagrams and sequence diagrams, we believe that the benefits are likely to out-weigh the increased complexity resulting from the proposed enhancements. The enhanced use case diagrams might also be used for communicating information system scope alternatives to the stakeholders and discussing associated costs and benefits.

Since our data was generated using our guidelines either partially (SIM treatment) or completely (ENH treatment), the statistical results support the potential benefits of using our proposed guidelines for converting activities identified in work system snapshots to enhanced use case diagrams. Further experimentation with these guidelines could improve the guidelines themselves, or could lead to other approaches to generate further insights during the process of creating use case diagrams.

\section{Limitations}

The experiment reported here has a number of limitations related to the data set size, use of student subjects, and use of a particular case study that may have biased the results in some way. Even with a small data set our study produced statistically significant results. Since we have analyzed the use case diagrams created by student teams rather than individual students, we believe that the resulting diagrams are comparable to the work produced by novice analysts in business situations. Another limitation of this study is related to the task that required creation of only one use case diagram for the information system. However, we believe that the method can be applied to larger systems that require several use case diagrams as commonly done in practice. Although supplementing use case diagrams with detailed narratives could have resulted in a better and more complete description of information system scope, it is not possible to incorporate such tasks in a typical laboratory experiment of 2 to 3 hours duration.

\section{Future Research}

The encouraging results from the current research can serve as the basis for future research in a number of directions. Similar experiments should be performed with different groups of subjects and with different case write-ups to verify that the initial results reported here are broadly applicable. It would be especially valuable to repeat several versions of the experiment with experienced systems analysts. The results with experienced systems analysts could demonstrate the benefits of using the work system snapshots, or could show that they already consider and incorporate the concepts that are captured in work system snapshots and related tools. Experiments with experienced systems analysis might also lead to new ways to use work system snapshots and/or related tools within the process of creating use case diagrams. 
Further research might also explore a more radical possibility of assuming that the work system snapshot and related tabular tools from the work system method might replace use cases, instead of serving as a starting point preceding the development of use cases. With that more radical approach, initial stages of the requirements analysis would focus the desired changes in the "as is" work system. Those changes could be summarized through the difference between work system snapshots for the "as is" and "to be" work systems. Once business and IT professionals have agreed on the desired directions for improvement in the work system, guidelines such as those suggested in section 3.1 could be used to create use cases that could then be used by IT professionals as the starting point for further analysis and design efforts based on UML. For this approach to be practical, it would be necessary to augment the work system snapshots with analysis tools that allow work system participants to understand more about the various special cases, exceptions, and important alternative flows that that they need to discuss among themselves and with IT professionals. On-going research in that direction, including new metamodels underlying the work system framework, is exploring the possibility of starting with work system snapshots, providing additional tools that specify information, technology, and resources at each step, and then proceeding with decomposition into subsystems of each work system.

In effect, the latter approach would question the fundamental idea of "use case" as the starting point for communication between business and IT professionals. For business professionals, the question of how to create and improve work systems is more important and more readily understandable than the question of how to identify the various use cases for a technical artifact that is being built. Regardless of whether it would prove useful to question the fundamental idea of use cases, it is possible that research about combining work system ideas with use case diagramming could lead to better modeling and better communication between business and IT professionals during systems analysis and design. 


\section{REFERENCES}

Ackoff, R. L. (1971). Towards a system of systems concepts. Management Science, 17(11), 661-671. doi:10.1287/ mnsc.17.11.661

Ackoff, R. L. (1973). Science in the systems age: Beyond IE, OR, and MS. Operations Research, 21(3), 661-671. doi:10.1287/opre.21.3.661

Alter, S. (2006). The work system method: connecting people, processes, and IT for business results. Work System Press.

Alter, S. (2008). Defining information systems as work systems: Implications for the IS field. European Journal of Information Systems, 17(5), 448-469. doi:10.1057/ejis.2008.37

Alter, S. (2013) Work System Theory: Overview of Core Concepts, Extensions, and Challenges for the Future, Journal of the Association for Information Systems, 14(2), 72-121.Anda, B., \& Sjoberg, D.I.K. (2002). Towards an inspection technique for use case models.Proceedings of the 14th International Conference on Software Engineering and Knowledge Engineering, Ischia, Italy (pp. 127-134).

Anton, A. I., Carter, R. A., Dagnino, A., Dempster, J. H., \& Siege, D. F. (2001). Deriving goals from a use-case based requirements specification. Requirements Engineering, 6(1), 63-73. doi:10.1007/PL00010356

Baekgaard, L. (2005, March 15-16). From Use Cases to Activity Cases. Proceedings of ALOIS`05, Limerick, Ireland 2005, P. J. Aggerfalk, L. Bannon, \& B. Fitzgerald (eds.)

Bolloju, N., \& Leung, F. S. K. (2006). Assisting novice analysts in developing quality conceptual models with UML. Communications of the ACM, 49(7), 108-112. doi:10.1145/1139922.1139926

Buschmann, F. (2009). Learning from Failure, Part 1: Scoping and Requirements Woes. IEEE Software, 26(6), 68-69. doi:10.1109/MS.2009.179

Checkland, P. (1999). Systems thinking, systems practice: includes a 30-year retrospective. Chichester, UK: John Wiley \& Sons.

Cheng, B. H. C., \& Atlee, J. M. (2007). Research directions in requirements engineering. Proceedings of Future of Software Engineering (pp. 285-303). doi:10.1109/FOSE.2007.17

Churchman, C. W. (1970). Operations research as a profession. Management Science, 17(2), 37-53. doi:10.1287/ mnsc.17.2.B37

Coad, P., North, D., \& Mayfield, M. (1995). Object models: strategies, patterns, applications. Upper Saddle River, NJ, USA: Yourdon Press.

Cockburn, A. (2001). Writing effective use cases. MA: Addison-Wesley Reading.

Dobing, B., \& Parsons, J. (2006, May01). Dobing, B. \& Parsons, J. (2006). How UML is used. Communications of the ACM, 49(5), 109-113. doi:10.1145/1125944.1125949

Dobing, B., \& Parsons, J. (2008). Dimensions of UML diagram use: A survey of practitioners. Journal of Database Management, 19(1), 1-18. doi:10.4018/jdm.2008010101

Eichelberger, H. (2008). Automatic layout of UML use case diagrams.Proceedings of the 4th ACM symposium on Software visualization (SoftVis '08)., 105-114. doi:10.1145/1409720.1409738

Erickson, J., \& Siau, K. (2007). Can UML Be Simplified? Practitioner Use of UML in Separate Domains in Proceedings of the Workshop on Exploring Modeling Methods for Systems Analysis and Design (EMMSAD'07), Trondheim, Norway, Tapir Academic Press, Trondheim, Norway, 87-96.

Eriksson, M., Börstler, J., \& Borg, K. (2005). The PLUSS Approach - Domain Modeling with Features, Use Cases and Use Case Realizations. In H. Obbink \& K. Pohl (Eds.), Software Product Lines (Vol. 3714, pp. 33-44). Berlin, Heidelberg: Springer Berlin Heidelberg. doi:10.1007/11554844_5

Gelbard, R., Teeni, D., \& Sade, M. (2010). Object-Oriented Analysis: Is It Just Theory? IEEE Software, 27(1), 64-71. doi:10.1109/MS.2009.151 
Gemino, A., \& Parker, D. (2009). Use Case Diagrams in Support of Use Case Modeling: Deriving Understanding from the Picture. Journal of Database Management, 20(1), 1-24. doi:10.4018/jdm.2009010101

Glinz, M. (2000). Problems and deficiencies of UML as a requirements specification language.Proceedings of the 10th International Workshop on Software Specification and Design, San Diego, CA (pp. 11-22). doi:10.1109/ IWSSD.2000.891122

Gottesdiener, E. (2002) Top Ten Ways Project Teams Misuse Use Cases - and How to Correct Them. IBM, Rational Edge. Retrieved from [REMOVED HYPERLINK FIELD]http://www.ibm.com/developerworks/rational/ library/content/RationalEdge/jul02/TopTenWaysJul02.pdf

Kim, J., Park, S., \& Sugarman, V. (2006). Improving use case driven analysis using goal and scenario authoring: A linguistics-based approach. Data \& Knowledge Engineering, 58(1), 21-46. doi:10.1016/j.datak.2005.05.006

Langlands, M., \& Edwards, C. (2009) Business vs. System Use Cases. The IIBA.org. Retrieved from http://www. theiiba.org/Content/NavigationMenu/Events/CurrentArticles1/Business_vs_SystemUseCases.pdf

Liu, X. M., \& Wyner, G. M. (2009). Coordination analysis: a method for deriving use cases from process dependencies.Proceedings of the 4th International Conference on Design Science Research in Information Systems and Technology, Philadelphia, Pennsylvania. doi:10.1145/1555619.1555642

Mustafa, B. A. (2010). Comparing the Effect of Use Case Format on End User Understanding of System Requirements. Journal of Information Technology Research, 3(4), 1-20. doi:10.4018/jitr.2010100101

Mylopoulos, J., Chung, L., Liao, S., Wang, H., \& Yu, E. (2001). Exploring alternatives during requirements analysis. IEEE Software, 18(1), 92-96. doi:10.1109/52.903174

Nuseibeh, B., \& Easterbrook, S. (2000). Requirements engineering: a roadmap.Proceedings of the Conference on the Future of Software Engineering (pp. 35-46).

Ramirez, A., Vanpeperstraete, P., Rueckert, A., Odutola, K., \& Bennett, J. (2007). ArgoUML User Manual: A tutorial and reference description. Tigris.org-Open Source Software Engineering Tools.

Rosenberg, D., \& Stephens, M. (2007). Use case driven object modeling with UML: theory and practice. Apress.

Rubinstein, D. (2007, March 1). Standish group report: There's less development chaos today. Software Development Times.

Truex, D., Alter, S., \& Long, C. (2010). Systems Analysis for Everyone Else: Empowering Business Professionals through a Systems Analysis Method that Fits Their Needs. Proceedings of the 18th European Conference on Information Systems, South Africa.

Truex, D., Lakew, N., Alter, S., \& Sarkar, S. (2011, October). Extending a Systems Analysis Method for Business Professionals. Proceedings of theEuropean Design Science Symposium, Leixlip, Ireland.

OMG Unified Modeling Language, (2010). Superstructure Version 2.3. Retrieved from http://www.omg.org/ spec/UML/2.3/Superstructure

Yang-Turner, F., \& Lau, L. (2011). Extending use case diagrams to support requirements discovery. Proceedings of theWorkshop on Requirements Engineering for Systems, Services and Systems-of-Systems (RESS) (pp. 32-35). doi:10.1109/RESS.2011.6043929 


\section{APPENDIX 1}

\section{Work Systems Framework and Snapshot}

The basic ideas of the work system approach have been presented in a number of times, e.g., Alter (2006; 2008; 2013). The basis of the approach is work system theory (Alter, 2013), which consists of three main components:

- The definition of work system (mentioned below),

- The work system framework (Figure 4), which identifies nine elements that should be considered in even a rudimentary understanding of a work system

- The work system life cycle model, which summarizes how work systems change over time, and which is not discussed here since the current focus is on summarizing a work system rather than explaining how it evolves over time.

A work system is a system in which human participants and/or machines perform work using information, technology, and other resources to produce products and/or services for internal or external customers. Typical business organizations contain work systems that procure materials from suppliers, produce products, deliver products to customers, find customers, create financial reports, hire employees, coordinate work across departments, and perform many other functions. Almost all significant work systems in business and governmental organizations rely on IT in order to operate efficiently and effectively.

Work system is a general case for thinking about systems within or across organizations. Special cases that inherit the body of knowledge from work systems in general include information systems in general (whose processes and activities are totally devoted to processing information), supply chains in general (which cross organizations), and use of ecommerce websites (self-service work systems). In this context, an entire ERP suite is infrastructure shared by multiple work systems, whereas programs used in a specific work system are part of the technology within the work system.

\section{Figure 4. The Work System Framework}

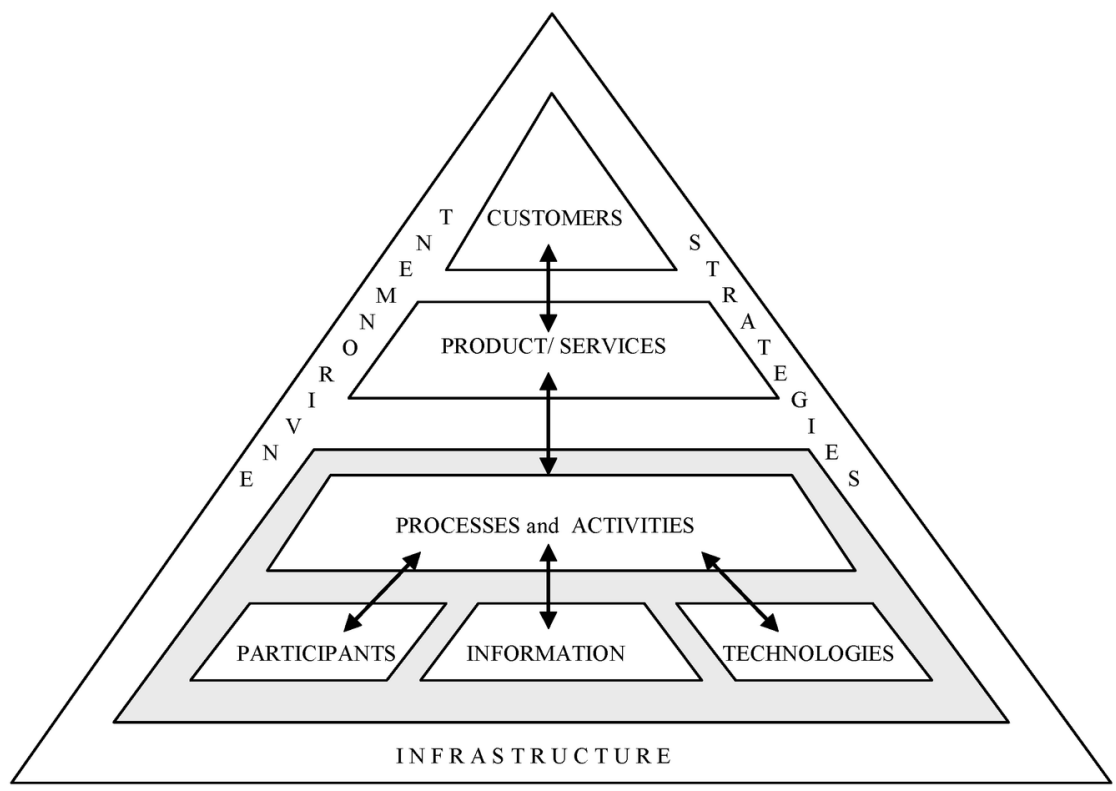


Work system framework. The nine elements of the work system framework are the basis for describing and analyzing an IT-reliant work system in an organization. The framework outlines a static view of a work system's form and function at a point in time and is designed to emphasize business rather than IT concerns. It covers situations that might or might not have a tightly defined business process and might or might not be IT-intensive. Careful definitions of each term presented in Alter (2013) provide clarifications such as the possibility that customers can also be participants (e.g., in ecommerce work systems) and the fact that processes and activities include both structured workflows and semi-structured routines.

Work system snapshot. A work system snapshot is a one-page summary of a work system (see Table 1 for an example) that identifies the main components of six central elements: customers, product/services, processes and activities, participants, information, and technologies. MBA and Executive MBA students have used this tool successfully in performing preliminary analyses of real world work systems in their own organizations (e.g., Truex et al., 2010). 


\section{APPENDIX 2}

\section{UBL Work Systems Snapshot}

\section{Table 4. UBL work system snapshot}

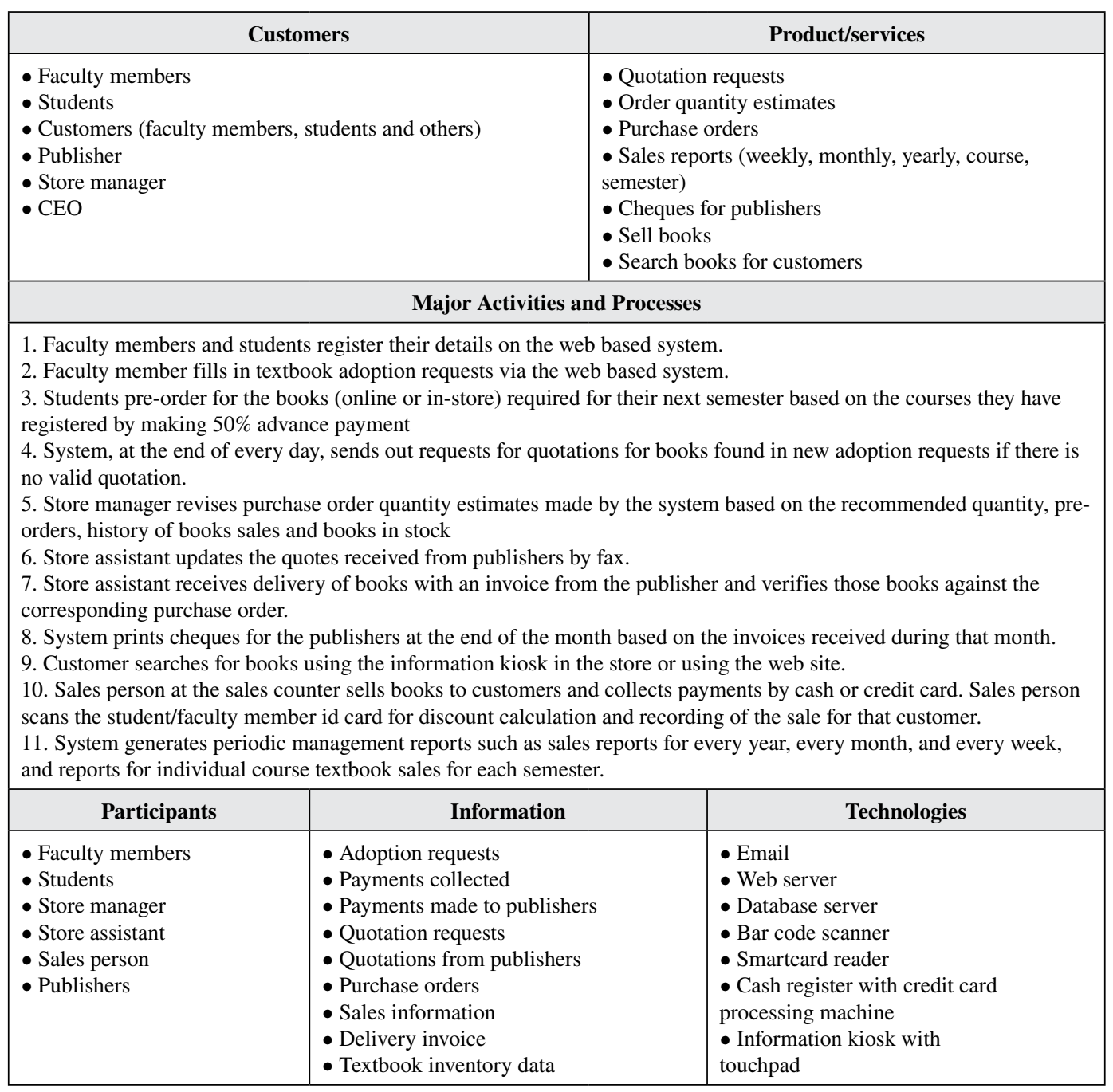




\section{APPENDIX 3}

\section{UBL System Feature List}

Overall Purpose Statement: To improve overall productivity of all staff, to enhance customer service, and to aid in decision making by senior management.

\section{Features for Conducting Business}

1. Capture textbook adoption requests from faculty members about 2 months before the beginning of each semester

2. Collect the textbook pre-orders from students one month before the beginning of each semester

3. Place purchase orders to publishers based on quotations received from publishers

4. Receive books delivered by publishers and verify against the purchase orders

5. Search book information by using ISBN, author name, title, and category, course codes, etc.

6. Handle sales at bookstore counters by shop assistants (may include discounts based on past transactions)

\section{Features for Analyzing Business Results}

1. Information about textbooks of different courses which are being sold below the expected level

2. Estimate order quantities for textbook based on sales history

3. Details of supply performance of the publishers (on time of delivery, sales volume, sales profit, late supplies and wrong supplies from publishers)

4. Generate yearly, monthly, and weekly sales reports for the management, as well as the individual course textbook sales for each semester

5. Identify the most profitable books or book categories (on sales volume, sales profit)

6. Generate reports on staff performance (on working hours, customer complaints, evaluation from supervisors, etc) 